\title{
Autoevaluación del conocimiento y aplicación del código de conducta por servidores públicos de salud en Tlaxcala
}

\section{Self-assessment of knowledge and application of the code of conduct by public health servers in Tlaxcala}

\author{
Óscar Castañeda Sánchez, ${ }^{*}$ Rosalba Jaramillo Sánchez **
}

https://doi.org/10.36105/mye.2022v33n1.06

\section{Resumen}

Introducción. El código de conducta establece pautas de comportamiento obligatorio para servidores públicos. Objetivo. Determinar el grado de conocimiento y aplicación del código de conducta por servidores públicos en salud. Métodos. Se realizó un estudio transversal descriptivo en servidores públicos del Seguro Social de Tlaxcala, incluyendo, a conveniencia, sus encuestas de autoevaluación del código de conducta. Variables. Categoría o puesto, antigüedad institucional y en puesto actual, grado de conocimiento y aplicación del código de conducta. Análisis de resultados con estadística descriptiva. Resultados. Se incluyeron 217 entrevistas de servidores públicos, con una antigüedad de $12.7 \pm$ 7.8 años, de los cuales el $15.2 \%$ corresponde a médicos no familiares. Conocimiento y aplicación del código: sobresaliente en el $49.8 \%$, notable en el $45.2 \%$, bueno en el $4.1 \%$ y suficiente en el $0.9 \%$. Conclusiones. La aplicación y conocimiento del código de

\footnotetext{
* Instituto Mexicano del Seguro Social de Tlaxcala, Tlaxcala. México. Correo electrónico: oscar.castanedasa@gmail.com https://orcid.org/0000-0002-9995-583X

** Instituto Mexicano del Seguro Social de Tlaxcala, Tlaxcala. México. Correo electrónico: rosalba.jaramillo@imss.gob.mx https://orcid.org/0000-0001-6828-7464 Recepción: 15 de agosto de 2021. Aceptación: 12 de septiembre de 2021.
}

Medicina y Ética - Enero-Marzo 2022 - Vol. 33 - Núm. 1 
conducta en 5 de cada 10 servidores públicos es sobresaliente, y notable en 4 de cada 10, incidiendo en la calidad de atención y servicio.

Palabras clave: personal de salud, principios éticos, deontología.

\section{Introducción}

La formación de buenos profesionales, con los conocimientos técnicos necesarios, no siempre conduce a la formación de profesionales «buenos» (1), pues se encuentran distorsiones relacionadas con los procesos de atención, a pesar de los códigos de cada profesional $(2,3)$. Ante la pretensión de cumplir con la legislación vigente y combatir la corrupción, un instrumento que puede beneficiar a las organizaciones es el código de conducta (4).

Se denomina código de conducta al compendio de normas o reglas desarrolladas dentro de un marco referencial para el correcto desenvolvimiento de las personas que forman parte de una organización (5), el cual sirve como un instrumento para aplicar las reglas éticas y morales en el actuar diario (6). Busca orientar las funciones directivas y operativas en un ambiente en el que se practiquen valores $(7,8)$, o cualidades descubiertas o construidas en las personas, tras la sucesión de acontecimientos, situaciones o cosas que merecen estimación, hasta convertirse en los ideales a partir de los cuales se regula la conducta $(9,10)$.

Las declaraciones normativas pueden referirse al empleo, condiciones de trabajo, respeto al medio ambiente, protección de datos, y pueden contener compromisos de la organización y de sus trabajadores para con terceros en cuanto a la calidad de los servicios y de la imagen corporativa (11-13). Así, un código de conducta es de carácter normativo y obligatorio para los miembros de una organización, y abarca obligaciones dentro de la esfera del deber y del gobernar (14). 
Autoevaluación del conocimiento y aplicación del código de conducta...

Para algunos autores, los códigos de conducta son un instrumento de gestión estratégica, que apoyan la toma de decisiones de las organizaciones e influyen en el comportamiento del personal directivo, y acaban impactando en la productividad de las empresas. Para otros, son instrumentos que dan a conocer las capacidades de la empresa y mejoran su imagen; no obstante, deben entenderse como complemento de la legislación de la institución $(15,16)$.

La implementación de un código de conducta integral marca el comportamiento que se espera recibir por parte de los profesionales de la salud, evitando actos que puedan perjudicar el comportamiento del equipo de la salud y una mala atención al paciente (17). Es fundamental para mantener una práctica o ejercicio profesional seguro (18); favorece la comunicación y el trabajo en equipo (19); establece los límites por los cuales la profesión ha de discurrir (20, $21)$; identifica las consecuencias que conlleva actuar de forma contraria; previene conflictos internos y externos (22) y celebra el desempeño de sus funciones (23), por lo cual más organizaciones optan por esta herramienta (24).

El Instituto Mexicano del Seguro Social (IMSS) cuenta con su Código de Conducta para los servidores públicos (25), el cual se difunde a todo el personal tanto directivo como operativo, esperando su aplicación. Este trabajo tiene como propósito, por tanto, determinar el grado de conocimiento y aplicación de dicho código de conducta por parte del servidor público del IMSS en Tlaxcala.

\section{Material y métodos}

Se realizó un estudio observacional, retrospectivo, retrolectivo, unicéntrico y homodémico, con diseño transversal descriptivo en el Órgano Operativo de Administración Desconcentrada del Seguro Social en Tlaxcala. Se incluyeron los resultados secundarios de la autoevaluación del conocimiento y de la aplicación del Código de Conducta realizada a los Servidores Públicos adscritos a la Jefatura

Medicina y Ética - Enero-Marzo 2022 - Vol. 33 - Núm. 1

https://doi.org/10.36105/mye.2022v33n1.06 
de Servicios de Prestaciones Médicas y a sus Unidades Médicas, como parte de la verificación de su cumplimiento, posterior a la difusión de la legislación institucional vigente. Para el tamaño de la muestra se tomó una confiabilidad del $99 \%$ y un margen de error del $10 \%$.

Las variables a estudiar corresponden al puesto o categoría, antigüedad institucional y antigüedad en el puesto actual, grado de conocimiento, resultado o nivel obtenido de la aplicación del instrumento de autoevaluación, y la aplicación o cumplimiento en el desempeño de las funciones de los once principios del Código de Conducta de los Servidores Públicos del Instituto Mexicano del Seguro Social: servicio, uso del cargo, uso y asignación de recursos, responsabilidad, honestidad, honradez, solidaridad, desarrollo profesional, manejo de información, equidad e imparcialidad y salud, seguridad y conservación del medio ambiente.

Para la recolección de los datos, se aplicó un formulario electrónico que constó de 86 reactivos: los primeros cinco recabaron datos laborales y de formación de los participantes; los siguientes cuatro abordaron las definiciones contenidas para el enfoque de los principios; dos con respuesta de opción múltiple y dos dicotómicas, con respuestas entre falso y verdadero. Los siguientes 77 reactivos corresponden a las acciones que se deben y que no se deben realizar en apego a los 11 principios, con respuestas dicotómicas (debo, no debo), los cuales fueron previamente revisados por un equipo de expertos y pilotado en un grupo de médicos internos.

Para determinar el nivel del grado de conocimiento y aplicación, se establecieron las siguientes puntuaciones y calificaciones o niveles, con base en el diferencial semántico y en la dimensión de evaluación: sobresaliente $=76-81$ puntos; notable $=70-75$ puntos; bueno $=64-69$ puntos; suficiente $=58-63$ puntos, $\mathrm{y}$ deficiente $=57$ puntos o menos.

El análisis de las variables se realizó a través del paquete estadístico de SPSS para Windows, con el cual se aplicaron frecuencias y 
Autoevaluación del conocimiento y aplicación del código de conducta...

porcentajes para las variables nominales y ordinales, y la media y la desviación estándar para las variables cuantitativas.

De acuerdo con el Reglamento de la Ley General de Salud en Materia de Investigación para la Salud, en su artículo 17, se considera como una investigación sin riesgo cuando el Jefe de Servicios de Prestaciones Médicas autoriza el uso de los resultados de las encuestas. El protocolo fue sometido al Comité Local de Investigación en Salud 2902 del Instituto Mexicano del Seguro Social, y fue aprobado con el número de registro R-2020-2902-003.

\section{Resultados}

Participaron 217 servidores públicos (Figura 1), con una antigüedad de $12.7 \pm 7.8$ años, y de $6.2 \pm 6.6$ años en el puesto actual. El mayor número de participantes corresponde a los médicos no familiares $(14.7 \%)$, seguido de los médicos familiares $(12.4 \%)$, auxiliares de enfermería general $(11.5 \%)$, directores de las unidades médicas $(7.8 \%)$, coordinadores clínicos de hospitales, al igual que coordinadores delegacionales $(5.1 \%)$. El resto corresponde a las de-

Figura 1. Unidad de adscripción de los servidores públicos.

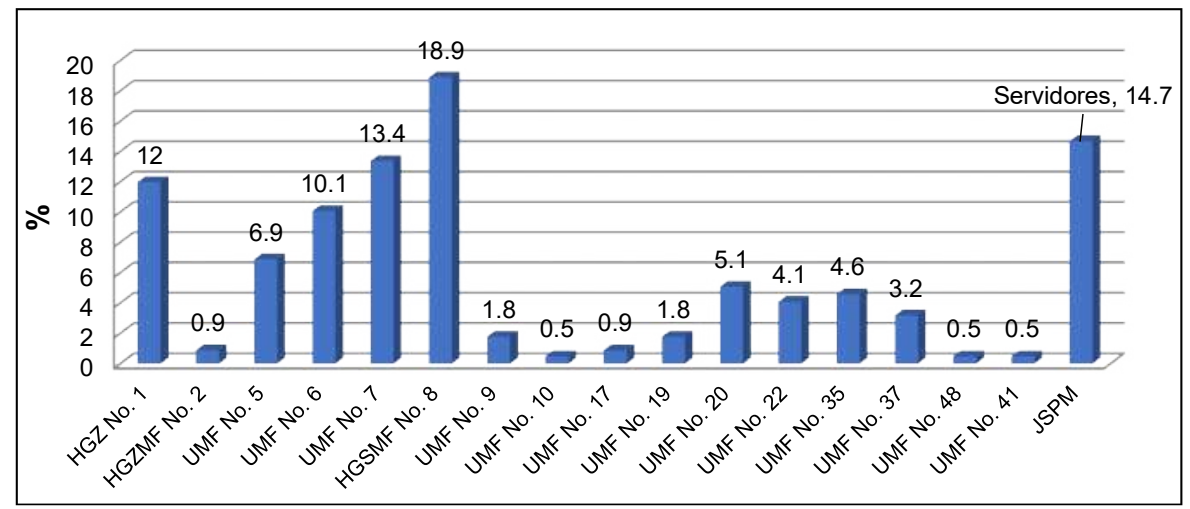

Fuente: Formularios Coordinación de Planeación y Enlace Institucional, IMSS Tlaxcala, 2019.

Medicina y Ética - Enero-Marzo 2022 - Vol. 33 - Núm. 1

https://doi.org/10.36105/mye.2022v33n1.06 
más categorías clínicas y no clínicas, como los funcionarios con más de 20 años de antigüedad por grupos de edad, el personal técnico de atención y orientación al derechohabiente, el personal auxiliar de almacén y el personal jefe de piso de enfermería. Por otro lado, el personal técnico de conservación, el personal auxiliar de limpieza e higiene, el personal operador de ambulancias y el personal auxiliar de oficina son los más jóvenes, con menos de 5 años de antigüedad (Figura 2).

En relación con las definiciones operacionales para la aplicación del Código de Conducta, el 50.9\% tienen conocimiento de lo que es el Código de Conducta; el 22.9\% de lo que es un servidor públi-

Figura 2. Servidores públicos por categoría y antigüedad.

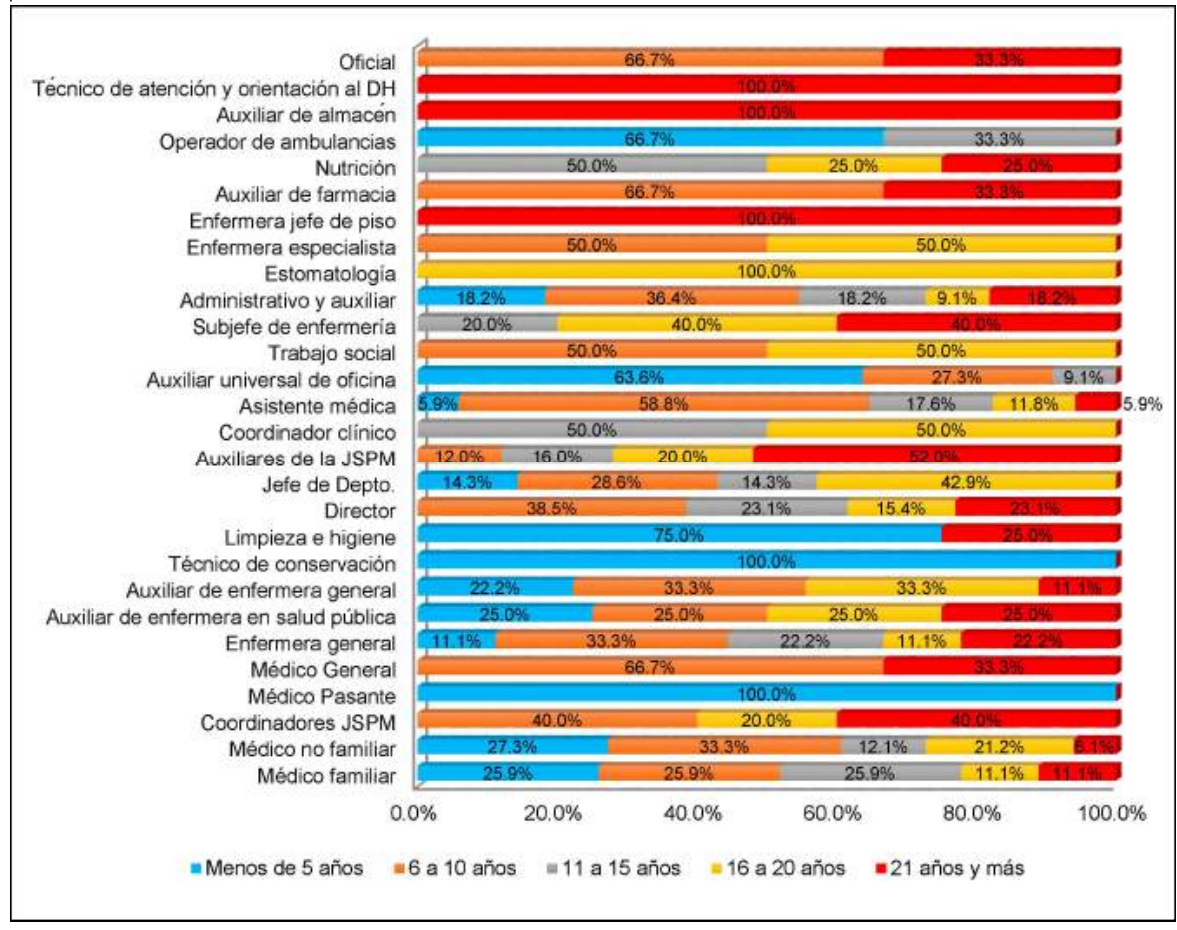

Fuente: Formularios Coordinación de Planeación y Enlace Institucional, IMSs Tlaxcala, 2019. 
Autoevaluación del conocimiento y aplicación del código de conducta...

co; el 100\% de lo que es un derechohabiente y el $92.1 \%$ de lo que es un usuario.

En relación con los valores, el nivel de conocimiento y aplicación fue sobresaliente en el caso de «disposición de servicio»; notable en el «uso del cargo público»; sobresaliente en el «uso y asignación de recursos», al igual que en «responsabilidad»; mientras tanto, en «honestidad» fue notable; sobresaliente en «honradez», así como en «solidaridad», «desarrollo profesional», «manejo de la información» y «equidad e imparcialidad». Finalmente, en el caso de «salud, seguridad y conservación del medio ambiente» fue notable, como puede observarse en la Figura 3.

Figura 3. Nivel de conocimiento y aplicación de los valores del Código de Conducta.

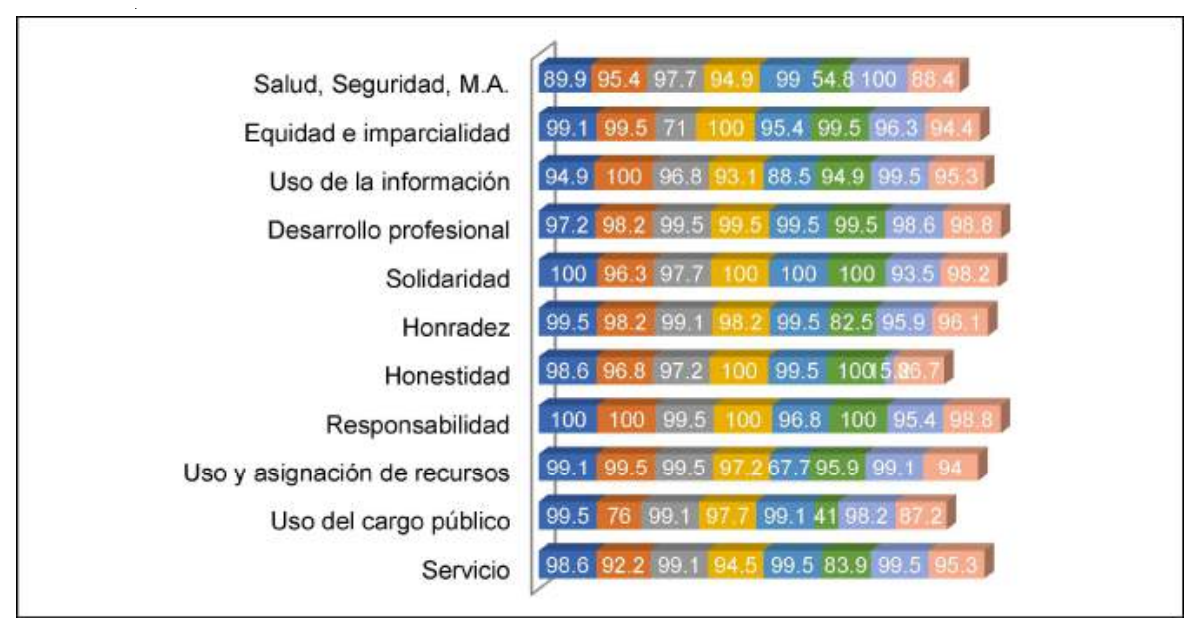

Fuente: Formularios Coordinación de Planeación y Enlace Institucional, IMSs Tlaxcala, 2019.

El conocimiento y el grado de aplicación del Código de Conducta se presentan en la Figura 4, y en la Figura 5 se puede observar por categorías.

Por grupos de antigüedad, el grupo de menos de 5 años tuvo un conocimiento y aplicación sobresaliente en el 25\%; notable en el 
Figura 4. Grado de conocimiento y aplicación del Código de Conducta.

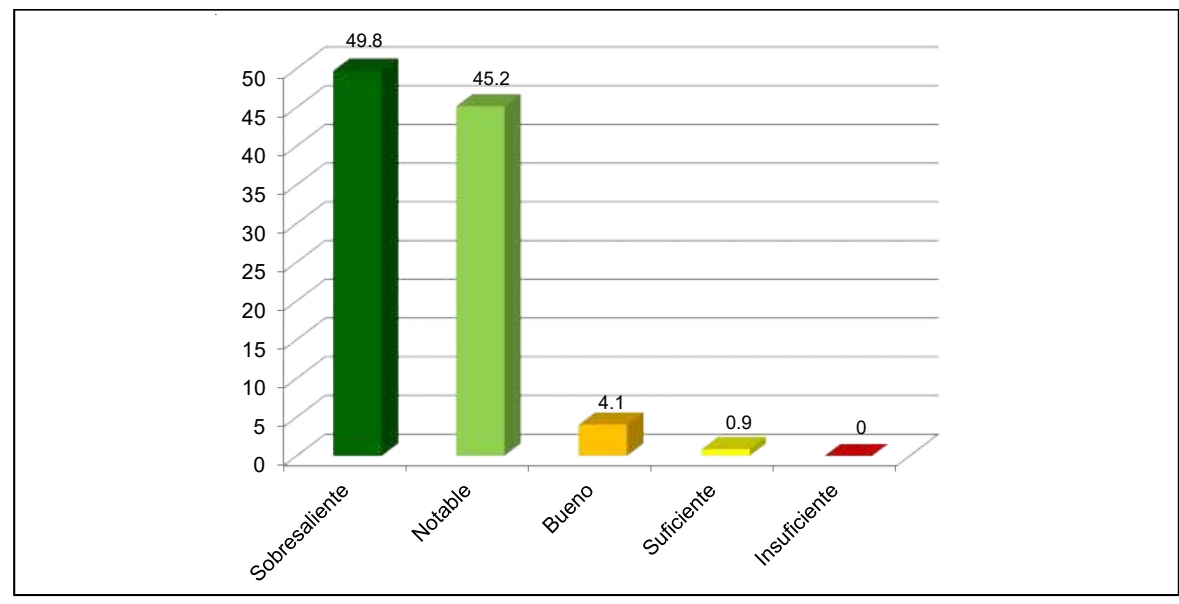

Fuente: Formularios Coordinación de Planeación y Enlace Institucional, IMSS Tlaxcala, 2019.

$65.9 \%$ y bueno en el $9.1 \%$. El grupo de 6 a 10 años obtuvo sobresaliente en el 58.1\% y notable en el $41.9 \%$. El grupo de 11 a 15 años, sobresaliente en el $60.6 \%$, notable en el $36.4 \%$ y bueno en el 3\%. Por su parte, el grupo de 16 a 20 años salió sobresaliente en el $56.8 \%$, notable en el $35.1 \%$, bueno en el $5.4 \%$ y suficiente en el $2.7 \%$. Por último, el grupo de 21 años y más obtuvo sobresaliente en el $48.8 \%$, notable en el $43.9 \%$, bueno en el $4.9 \%$ y suficiente en el $2.4 \%$.

\section{Discusión}

De acuerdo con Vargas (2015) (26), una administración pública orientada a servir al público o al colectivo como origen de su desempeño, obliga a repensar y a rediseñar sus funciones y procesos bajo nuevas formas de organización que propicien la transparencia y rendición de cuentas y tome en consideración las opiniones de los usuarios de los servicios, involucrándolos, además, en la toma de decisiones y en los procesos para su atención. Para ello, se requiere la im- 
Autoevaluación del conocimiento y aplicación del código de conducta...

Figura 5. Nivel de conocimiento y aplicación del Código de Conducta por categorías.

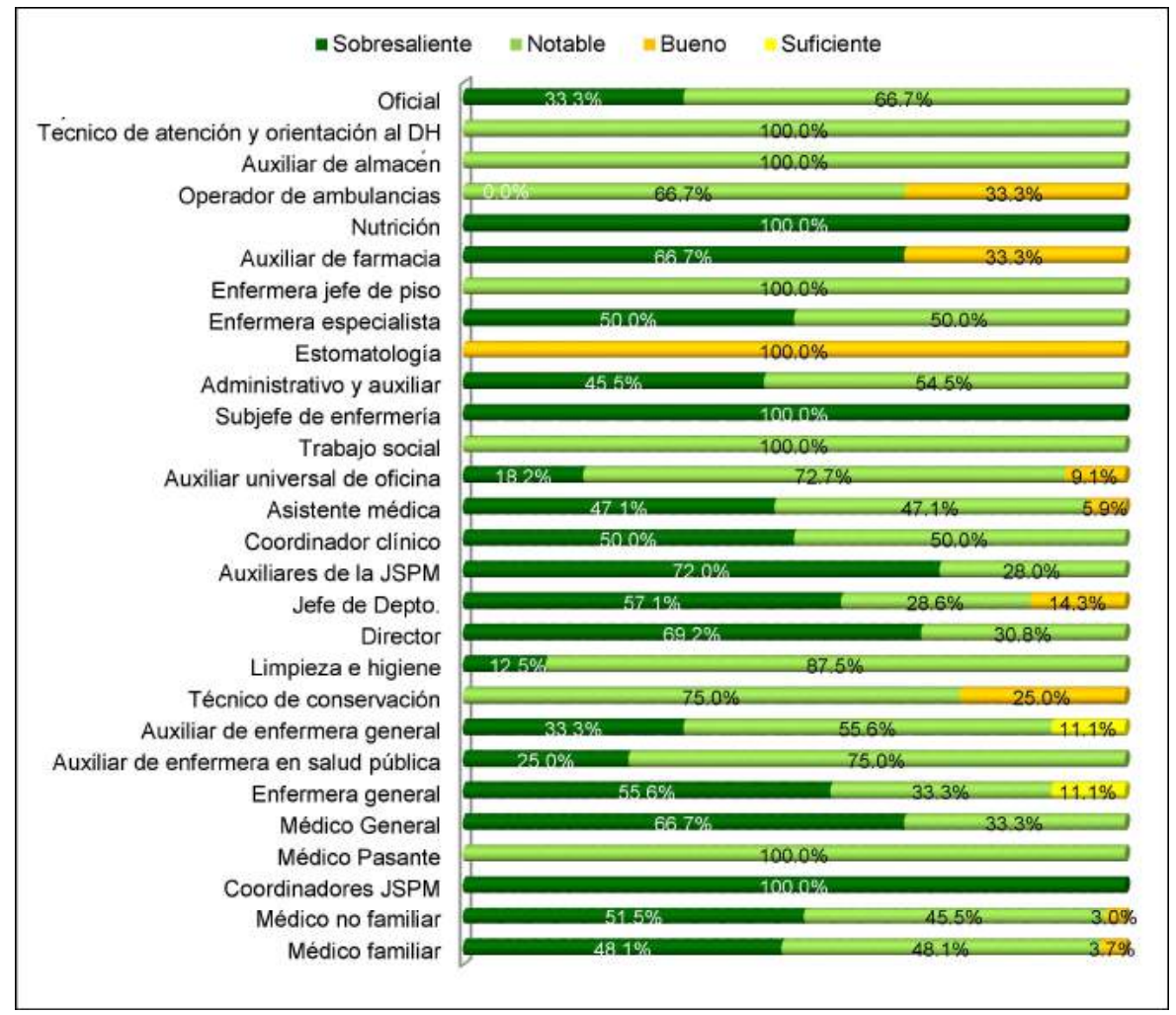

Fuente: Formularios Coordinación de Planeación y Enlace Institucional, IMSS Tlaxcala, 2019.

plementación de códigos dirigidos a los servidores públicos con el establecimiento de valores que favorezcan un desempeño eficiente en un ambiente armónico.

Para garantizar el cumplimiento de los principios de legalidad, honradez, lealtad, imparcialidad y eficiencia que rigen el servicio público, emanados del Código de Ética de los Servidores Públicos de la Administración Pública Federal, publicado en el DOF el 31 de julio del 2002, se establece el Código de Conducta, el cual tiene como finalidad delimitar la actuación que deben observar los servi-

Medicina y Ética - Enero-Marzo 2022 - Vol. 33 - Núm. 1 
dores públicos del Instituto Mexicano del Seguro Social (IMSS), en el desempeño de sus funciones (25). Dichos documentos regularmente son difundidos a los trabajadores o funcionarios sin evaluar su aplicación o el conocimiento de cómo actuar durante su desempeño; es decir, su conducta organizacional, como la define Pérez Zepeda (2016) (5).

Ahora, como comentan Gómez y Delgado (2018) (6), el Código de Conducta no sólo se enfoca al personal clínico, sino que también va dirigido a regular la forma de actuar durante el desempeño del personal no clínico. No obstante, los estudios al respecto son escasos. Authement (2017) (17), tras la aplicación de un Código de Conducta en una escuela de enfermería, observó una disminución del $25 \%$ de actos de comportamiento incivil, pero no evaluó el grado de conocimiento del Código por parte de los alumnos.

En otro estudio realizado por Parra et al. (2017) (20) en personal de enfermería, se evaluó la aplicación de su Código Deontológico, y se reportó que 8 de cada 10 funcionarios aplican sus principios. En el caso de este trabajo y con respecto al personal de enfermería, 9 de cada 10 tienen un conocimiento y aplicación aceptable de los valores del Código de Conducta. Entre las enfermeras especialistas, 10 de cada 10, y entre las generales y auxiliares, 9 de cada 10 .

En el área médica no se encuentran evaluaciones de la aplicación de los valores organizacionales; sólo revisiones de sus principios deontológicos. La adopción de un modelo norteamericano está fuera de sintonía con las necesidades latinoamericanas, cuyos presupuestos teóricos, así como la pluralidad de sujetos morales y las prácticas multi, inter y transdisciplinarias orientadas a la salud pública y colectiva y a la defensa de los más vulnerables (2) encuentran en el médico especialista un conocimiento y aplicación del Código de Conducta de 9 de cada 10, frente al 100\% de los médicos generales.

En el caso de estomatología, los estudios encontrados abordan la aplicación de los valores y/o principios deontológicos en los estudiantes, entre los que se observó una aplicación en 6 de cada 10 
Autoevaluación del conocimiento y aplicación del código de conducta...

alumnos (9). Con base en los resultados de este trabajo, el 100\% de los estomatólogos obtuvieron una aplicación y conocimiento buenos del Código de conducta; es decir, se quedaron en la media.

En el resto de los servicios auxiliares y de apoyo, en concreto en el caso de los paraclínicos, el conocimiento y la aplicación va de notable a sobresaliente, mientras que en los técnicos sólo es buena en 1-3 de cada 10.

Por grupos de edad, los grupos intermedios, o sea, los que van de 6 a 10 años y de 11 a 15, muestran un mejor conocimiento y aplicación del Código de Conducta que los extremos. No obstante, el grupo intermedio de 11 a 15 años de antigüedad es el más sobresaliente.

De acuerdo con Arroyo Chacón (2018) (22), en los Códigos de Conducta se presentan cuatro tipos de comportamientos de observancia: las conductas obligatorias, las óptimas, las deseables y las prohibidas. En el caso del Código de Conducta del IMSS, las primeras tres se abordan como «el debo», y la última, como el «no debo». $\mathrm{Al}$ respecto, a pesar de que el conocimiento y aplicación en general va de notable a sobresaliente, encontramos áreas de oportunidad, tanto en las conductas que se deben emprender, como en aquellas que no se deben realizar en el ejercicio de las funciones.

Por otra parte, en aquellos valores con resultados más bajos destacan algunas actividades de honestidad, uso del cargo, servicio, uso y asignación de recursos, honradez, manejo de la información, salud, seguridad y conservación del medio ambiente; sin embargo, las fortalezas son sobresalientes en los valores de responsabilidad, honestidad, solidaridad, manejo de la información y salud, seguridad y conservación del medio ambiente.

Como debilidad del estudio se puede comentar la búsqueda de la escolaridad de los participantes. No obstante, en algunos casos puede determinarse de acuerdo con la categoría. Por otro lado, no se identificó el sexo, para no incurrir en situaciones que puedan marcar discriminación, conforme a la NOM-R-025-SCFI-2015 sobre igualdad laboral y no discriminación. Sin embargo, este estudio

Medicina y Ética - Enero-Marzo 2022 - Vol. 33 - Núm. 1

https://doi.org/10.36105/mye.2022v33n1.06 
puede ser punto de partida para otras investigaciones relacionadas en materia de aplicación deontológica y su impacto en el desempeño de las funciones del personal de salud.

Finalmente, entre los comentarios que realizaron los participantes al ejercicio de la evaluación, está el reconocimiento de contar con un Código de Conducta y el hecho de realizar este tipo de autoevaluaciones, sugiriendo que se realicen de forma frecuente e incluso que se editen videos y se hagan aplicaciones para su revisión constante.

\section{Conclusiones}

La aplicación y conocimiento del Código de Conducta es sobresaliente en 5 de cada 10 trabajadores, y notable en 4 de cada 10 . La responsabilidad y el desarrollo profesional son los valores más destacados, mientras que la honestidad, el uso del cargo público y la salud, la seguridad y conservación del medio ambiente son los más débiles. Los coordinadores de la Jefatura de Servicios de Prestaciones Médicas, los subjefes de enfermería y el personal de nutrición muestran un conocimiento y aplicación sobresaliente del Código, entre los que destacan 6 de cada 10 funcionarios del grupo de 6 a 10 años de antigüedad. Esta situación incide en la calidad de la atención con la que se otorgan los servicios prestados, tanto en áreas clínicas, como en no clínicas.

\section{Referencias bibliográficas}

1. Pérez Romero LA, Garzón Castrillón MA, Ibarra Mares A. Código de ética empresarial para las Pymes: marco de referencia para la sostenibilidad y responsabilidad social empresarial (RSE). Espacios. 2015; 36(2): 11. https://www.revistaespacios.com/a15v36n02/15360211.html 2. Passos Soares FJ, Eri Shimizu H, Garrafa V. Código de ética médica brasileño: límites deontológicos y bioéticos. Rev Biot. 2017; 25(2): 244-254. 
Autoevaluación del conocimiento y aplicación del código de conducta...

http://www.redalyc.org/articulo.oa?id=361552153005

https://doi.org/10.1590/1983-80422017252184

3. Román Collazo CA, Urgilez Pesantez PC, Andrade Campoverde DP. Consideraciones éticas en la dispensación de medicamentos en farmacias de Ecuador. Rev Habanera Cienc Medi. 2020; 19(5): 76-92. http://www.revhabanera.sld.cu/ index.php/rhab/article/view/2954

4. Oliveira Araujo A. Análisis del código de conducta de las instituciones financieras de la BM\&FBovespa, a la luz de las recomendaciones del instituto brasileño del gobierno corporativo. RCC. 2018; 15(34): 115-143.

https://doi.org/10.5007/2175-8069.2018v15n34p115

5. Pérez Zepeda M. La conducta organizacional. ¿Es necesario instaurar el código de conducta organizacional? Revista Empresarial. 2016; 10(37): 21-26.

https://dialnet.unirioja.es/servlet/articulo?codigo $=5580336$

6. Gómez PC, Delgado Marroquín MT. Las relaciones entre profesionales en los códigos de deontología de diversas profesiones de la salud. Cuad Bioét. 2018; 29(97): 271-280. https://doi.org/10.30444/CB.11

7. Martínez Negrete E. Hacia un código de ética para la educación virtual, basado en las cinco mentes de futuro. Rev Mex Bachill Distancia. 2014; 6(11): 127-134. https://doi.org/10.22201/cuaed.20074751e.2014.11.65017

8. Hupffer HM, Benemann C. Autonomía de la relación médico-paciente en el aspecto de atención paliativa: un estudio en los códigos de conducta médica en Brasil y Portugal. Rev Praxis. 2019; 2: 338-363.

https://doi.org/10.25112/rpr.v2i0.1760

9. Palomer L, López R. Medición de los valores éticos y morales enseñados en la carrera de odontología de la Pontificia Universidad Católica de Chile, desde la apreciación docente. FEM. 2016; 19(2): 77-84.

https://doi.org/10.33588/fem.192.832

10. Arrojo MJ. Valores éticos y cambio tecnológico en la comunicación audiovisual: de la ciencia a la tecnología. Palabra Clave. 2019; 22(1): 1-33.

https://doi.org/10.5294/pacla.2019.22.1.8

11. Campos Victoria I. Despido disciplinario e incumplimiento del código de conducta de la empresa. Comentario a la sentencia del TSJ de Castilla La Mancha, de 9 de febrero de 2017. IUSLabor. 2017; 2: 1-15. https://raco.cat/index.php/IUSLabor /article/view/333009

12. Cifuentes Muñoz A. Epistemología implícita en el código de ética profesional del colegio de psicólogos de Chile. Cinta Moebio. 2019; 64: 51-57.

https://doi.org/10.4067/s0717-554x2019000100051

13. Ramos Serpa G, López Falcón A. Formación ética del profesional y ética profesional del docente. Estud Pedagog. 2019; 45(3): 185-199.

https://doi.org/10.4067/S0718-07052019000300185

14. Barreda Hernández D, Mulet Alberola A, González Bermejo D, Soler Company E. El porqué de un código de ética farmacéutico: Código Español de Ética Farmacéutica. Farm Hosp. 2017; 41(3): 401-409.

https://doi.org/10.7399/fh.2017.41.3.10611

Medicina y Ética - Enero-Marzo 2022 - Vol. 33 - Núm. 1 
15. Biedma Ferrer JM. Los códigos de conducta en las organizaciones. Estudio del compromiso de los códigos de conducta con los recursos humanos en las organizaciones turísticas. Turydes. (Consultado el 23 de mayo de 2021). 2018; 24. Disponible en: https://www.eumed.net/rev/turydes/24/codigo-conducta.html 16. Parra DI, Peñaloza Jaimes SD, Cárdenas Villabona MV, De Cruz NR, Amaya Díaz HC, Arboleda de Pérez LB et al. Responsabilidades éticas en la práctica de enfermería en instituciones de alta complejidad. Rev Cuid. 2019; 10(3): 1-16. https://doi.org/10.15649/cuidarte.v10i3.662

17. Authement R. ¿Puede un código de conducta integral combatir la incivilidad de los estudiantes de enfermería? Nursing. 2017; 34(4): 60-64. https://doi.org/10.1016/j.nursi.2017.07.017

18. Cowin LS, Riley TK, Heiler J, Gregory LR. La relevancia del código de conducta de enfermeras y matronas en Australia. Int Nurs Rev. 2019; 66(3): 343-352. https://dialnet.unirioja.es/servlet/articulo?codigo $=7589672$ 19. Nieto Jiménez JC. La transparencia de las Cortes Generales a la luz de las recomendaciones del GRECO: el Código de Conducta de los Diputados. Rev Esp Transp. 2020; 10: 31-47. https://dialnet.unirioja.es/servlet/articulo?codigo=75917

20. Parra DI, Rey de Cruz N, Amaya Díaz HC, Viviana Cárdenas M, Arboleda LB, Corredor Jurado Y, et al. Percepción de las enfermeras sobre la aplicación del código deontológico de enfermería en Colombia. Rev Cuid. 2016; 7(2): 1310-1317. https://doi.org/10.15649/cuidarte.v7i2.335

21. Faralli $C$. Normas éticas y normas jurídicas en la relación médico-paciente. TELOS. 2016; 18(3): 399-414. https://www.redalyc.org/pdf/993/99346931004.pdf

22. Arroyo Chacón JI. Los códigos de ética y los códigos de conducta en la promoción de la ética organizacional. Rev Nac Adm. 2018; 9(1): 87-103.

https://doi.org/10.22458/rna.v9i1.2104

23. Alonso Pérez MT. El código de buenas prácticas de reestructuración viable de las deudas con garantía hipotecaria sobre la vivienda habitual. Rev Derecho Civ. 2015; 2(2): 31-67. https://www.nreg.es/ojs/index.php/RDC/article/view/108

24. Arredondo Trapero FG, Villa Castaño LE, De la Garza García J. Propuesta para el diseño de un código de ética empresarial basado en la ética kantiana. Cuad Adm. 2014; 30(52): 9-19.

https://www.redalyc.org/pdf/2250/225033236002.pdf

25. Código de Conducta de las y los Servidores Públicos del Instituto Mexicano del Seguro Social. Publicado en el Diario Oficial de la Federación de los Estados Unidos Mexicanos, Oficio Circular No. SP/100/0762/02 (31 de julio de 2002).

26. Vargas Hernández JG. Implicaciones del nuevo generalismo en los valores éticos, democráticos y profesionales del servidor público. Espacios Públicos. 2016; 19(46): 51-72. https://www.redalyc.org/pdf/676/67648385003.pdf

Esta obra está bajo licencia internacional Creative Commons Reconocimiento-NoComercial-Compartirlgual 4.0. 